\title{
Astronomy, Physics, and Chemistry of $\mathbf{H}_{3}^{+}$
}

\author{
Takeshi Oka \\ Department of Astronomy and Astrophysics, and Department of \\ Chemistry, The Enrico Fermi Institute, The University of Chicago
}

\begin{abstract}
This is a report of a Discussion Meeting titled "Astronomy, Physics, and Chemistry of $\mathrm{H}_{3}^{+}$" which was held on February 9 and 10, 2000 at the Royal Society in London.
\end{abstract}

\section{Introduction}

$\mathrm{H}_{3}^{+}$is the simplest polyatomic system and is the most abundantly produced ion in molecular hydrogen dominated plasmas. It plays a pivotal role as the universal proton donor in the cosmic ray driven ion-neutral reaction scheme for the formation of interstellar molecules. The recent observations of the infrared spectrum of $\mathrm{H}_{3}^{+}$in dense molecular clouds (Geballe and Oka 1996; McCall et al. 1999) as well as in the diffuse interstellar medium (McCall et al. 1998; Geballe et al. 1999) have demonstrated the ubiquity of this fundamental molecular ion in interstellar space. The abundance of $\mathrm{H}_{3}^{+}$in molecular clouds had been anticipated but its abundance in the diffuse medium was surprising.

The spectrum has also been observed in outer planets as strong and pure emission and has become a powerful probe for monitoring morphology of plasma activities in planetary ionospheres, providing means for studying their magnetospheres from ground based observatories (Connerney and Satoh 2000).

These astronomical observations and their analysis have introduced novel questions whose solution require participation of researchers in different fields. In particular, the observed unexpectedly large column densities of $\mathrm{H}_{3}^{+}$(4-2 $\times$ $10^{14} \mathrm{~cm}^{-2}$ ) in diffuse clouds toward Cygnus OB2 No. 12 and 5 have revealed a major problem involving the dissociative recombination rate of $\mathrm{H}_{3}^{+}$. In both clouds $\mathrm{H}_{3}^{+}$is produced by cosmic ray ionization of $\mathrm{H}_{2}$ followed by the ion-neutral reaction $\mathrm{H}_{2}+\mathrm{H}_{2}^{+} \rightarrow \mathrm{H}_{3}^{+}+\mathrm{H}$. But the destruction of $\mathrm{H}_{3}^{+}$in diffuse clouds (due to electron recombination) is supposed to be faster than that in dense clouds (due to proton hop reaction to $\mathrm{CO}$ ) by 2 to 3 orders of magnitude. In order to explain the comparable $\mathrm{H}_{3}^{+}$column densities observed in dense and diffuse clouds, we need to assume extraordinarily large diffuse clouds.

The Royal Society Discussion Meeting titled "Astronomy, Physics, and Chemistry of $\mathrm{H}_{3}^{+}$" was organized by E. Herbst, S. Miller, T. Oka, and J. K. G. Watson on February 9 and 10, 2000 in London where chemists, physicists, and astronomers discussed a variety of general and specific problems on $\mathrm{H}_{3}^{+}$from different perspectives. The fundamental nature of the molecular ion made such interdisciplinary discussions possible and fruitful. 


\section{Program}

The topics and discussion leaders (in parentheses) and the speakers and titles of talks are given below. The whole program including a poster session can be viewed on webpage, http://h3plus .uchicago.edu.

1. Spectroscopy (W. Klemperer, A. Carrington)

J. K. G. Watson, An introduction to the Spectroscopy of $\mathrm{H}_{3}^{+}$

B. J. McCall, Laboratory Spectroscopy of $\mathrm{H}_{3}^{+}$

I. $\mathrm{R}$. McNab, The near dissociation spectrum of $\mathrm{H}_{3}^{+}$

J. Tennyson, The theory of $\mathrm{H}_{3}^{+}$near its dissociation

2. Dynamics (D. Smith, D. Clary)

M. Larsson, Experimental studies of the dissociative recombination of $\mathrm{H}_{3}^{+}$

A. Suzor-Weiner, Dissociative recombination of $\mathrm{H}_{3}^{+}$: progress in theory

T. Amano, Infrared absorption spectroscopy of $\mathrm{D}_{3}$ : an investigation into the mechanism of triatomic hydrogen species

3. Planetary (R. Prangé)

J. E. P. Connerney, The $\mathrm{H}_{3}^{+}$ion: a remote diagnostics of the jovian magnetosphere

S. Miller, The role of $\mathrm{H}_{3}^{+}$in planetary atmosphere

4. Interstellar $\mathrm{H}_{3}^{+}$(E. F. van Dishoeck, D. A. Williams)

T. R. Geballe, $\mathrm{H}_{3}^{+}$between the stars

J. H. Black, The abundance and excitation of interstellar $\mathrm{H}_{3}^{+}$

E. Herbst, The astrochemistry of $\mathrm{H}_{3}^{+}$

T. J. Millar, The role of $\mathrm{H}_{2} \mathrm{D}^{+}$in the deuteration of interstellar molecules

E. Roueff, $\mathrm{H}_{3}^{+}$recombination and bistability in the interstellar medium

The Discussion Meeting was accompanied by two related meetings at University College London, "Molecules and Dust in Region of High Extinction" on February 8 and " $\mathrm{H}_{3}^{+}$, Further Discussions and Future Perspectives" on February 11. In the latter meeting findings obtained after the programming of the main meeting and some more speculative thoughts on various aspects of $\mathrm{H}_{3}^{+}$were presented. The speakers and titles of the meeting were as follows.

C. Cecchi-Pestellini, $\mathrm{H}_{3}^{+}$in diffuse interstellar clouds

(R. Stark), Detection of interstellar $\mathrm{H}_{2} \mathrm{D}^{+}$and its unique role in the chemistry of star formation

(M. Mumma), $\mathrm{H}_{3}^{+}$emission from exoplanets

R. Jaquet, Investigation of $\mathrm{H}_{3}^{+}$based on explicitly correlated wavefunctions

R. Johnsen, Review of the experimental work on the recombination of $\mathrm{H}_{3}^{+}$

D. Gerlich, Experimental studies of ion molecule reactions at low energies: fast and slow reactive ways from $\mathrm{H}^{+}$and $\mathrm{H}_{2}^{+}$to $\mathrm{H}_{3}^{+}$

A. Bandrauk, $\mathrm{H}_{3}^{+}$in intense laser fields-enhanced ionization, Coulomb explośion and harmonic generation

M. Cordonnier, Selection rules for ortho- and para- $\mathrm{H}_{3}^{+}$in ion-neutral reactions.

\section{Summary}

The laboratory spectroscopy is approaching high vibrational states near the top of the barrier to linearity (McCall 2000). A hyperspherical coordinate formalism 
is being developed to cope with the singularity at such point (Watson 2000). The highly congested $\mathrm{H}_{3}^{+}$spectrum near the dissociation limit (Kemp et al. 2000) presents a great challenge to theory (Tennyson et al. 2000).

Despite many experimental efforts, consensus has not been reached on the recombination rate of $\mathrm{H}_{3}^{+}$(Larsson 2000). The theoretical value differs from the experimental by more than orders of magnitude (Orel et al. 2000). This is a crucial problem for the analysis of interstellar $\mathrm{H}_{3}^{+}$. Spectroscopy of $\mathrm{D}_{3}$ provides insight for the formation of triatomic hydrogen (Amano \& Chan 2000).

Jovian magnetosphere has been studied by $\mathrm{H}_{3}^{+}$spectrum (Connerney and Satoh 2000). It was proposed (Miller et al. 2000) that $\mathrm{H}_{3}^{+}$also plays a major role in energy budget and dynamics of planetary atmospheres. It was speculated that $\mathrm{H}_{3}^{+}$emission might just be detectable from exoplanets using current technology.

The $\mathrm{H}_{3}^{+}$infrared absorption observed in molecular clouds provides a unique means to determine the basic parameters of the cloud. Observation of $\mathrm{H}_{3}^{+}$in diffuse clouds have been a surprise and its true implication is yet to be understood (Geballe 2000). Some models have been proposed (Black 2000; Cecchi-Pestellini and Dalgarno 2000). $\mathrm{H}_{3}^{+}$plays the central role in molecular formation (Herbst 2000), the high deuterium fractionation (Millar et al. 2000) and the bistability in model calculation (Pineau des Forêts and Roueff 2000).

The proceedings of the meeting has been published in the Philosophical Transactions of the Royal Society of London A358 (2000) and as a book.

\section{References}

Amano, T. \& Chan, M.-C. 2000, Phil. Trans. R. Soc. Lond., A358, 2457

Black, J. H. 2000, Phil. Trans. R. Soc. Lond., A358, 2515

Cecchi-Pestellini, C. \& Dalgarno, A. 2000, MNRAS, 313, L6

Connerney, J. E. P. \& Satoh, T. 2000, Phil. Trans. R. Soc. Lond., A358, 2471

Geballe, T. R. 2000, Phil. Trans. R. Soc. Lond., A358, 2503

Geballe, T. R. \& Oka, T. 1996, Nature, 384, 33

Geballe, T. R., McCall, B. J., Hinkle, K. H., \& Oka, T. 1999, ApJ, 510, 251

Herbst, E. 2000, Phil. Trans. R. Soc. Lond. A358, 2523

Kemp, F., et al. 2000, Phil. Trans. R. Soc. Lond. A358, 2403

Larsson, M. 2000, Phil. Trans. R. Soc. Lond. A358, 2433

McCall, B. J. 2000, Phil. Trans. R. Soc. Lond. A358, 2385

McCall, B. J., Geballe, T. R., Hinkle, K. H., \& Oka, T. 1998, Science, 279, 1910

McCall, B. J., Geballe, T. R., Hinkle, K. H., \& Oka, T. 1999, ApJ, 522, 3389

Millar, T. et al. 2000, Phil. Trans. R. Soc. A358, 2539

Miller, S., et al. 2000, Phil. Trans. R. Soc. Lond., A358, 2485

Orel, A. E., et al. 2000, Phil. Trans. R. Soc. Lond., A358, 2445

Pineau des Forêts, G. \& Roueff, E. 2000, Phil. Trans. R. Soc. Lond., A358, 2549

Tennyson, J., et al. 2000, Phil. Trans. R. Soc. Lond., A358, 2419

Watson, J. K. G. 2000, Phil. Trans. R. Soc. Lond., A358, 2371 